\title{
Cholesterol-Phospholipid Complexation in Fluid Bilayers as Evidenced by Nearest-Neighbor Recognition Measurements
}

\author{
Jianbing Zhang, Honghua Cao, and Steven L. Regen \\ Department of Chemistry, Lehigh University, Bethlehem, Pennsylvania 18015, E-mail: \\ slr0@lehigh.edu
}

\begin{abstract}
Nearest-neighbor recognition experiments have been carried out using varying ratios of exchangeable dimer analogs of 1,2-dimyristoyl-sn-glycero-3-phosphatidylglycerol and 1,2-distearoyl-snglycero-3-phosphatidylglycerol in cholesterol-rich unilamellar vesicles at $60^{\circ} \mathrm{C}$. Equilibrium dimer distributions that were obtained support a structural model of the liquid-ordered bilayer in which free cholesterol and the longer-chain phospholipid homodimer are in equilibrium with a complex of unique stoichiometry, where one cholesterol molecule combines with two of the long-chain phospholipid homodimers. In this model, the mixing of the short-chain with the uncomplexed longchain phospholipids is ideal, and the complexed dimers are shielded from the disulfide exchange reaction.
\end{abstract}

\section{Introduction}

The hypothesis that cholesterol associates with high-melting lipids to form liquid-ordered regions in cell membranes, and that these regions play a major role in signal transduction and membrane trafficking, has been the subject of considerable debate in recent years. ${ }^{1-4}$ This proposal has also stimulated efforts aimed at obtaining a deeper understanding of cholesterolphospholipid interactions in model systems. ${ }^{5-11}$ One of the most difficult issues, in this regard, relates to supramolecular structure. In particular, it remains to be established whether cholesterol-rich fluid bilayers that contain high-melting phospholipids above their main phase transition temperature exist as (i) a mixture of liquid-disordered and liquid-ordered phases (the latter are characterized by acyl chains that are as tightly packed as in the gel phase, but with a higher mobility due to the intercalated cholesterol), (ii) a single phase composed of complexed and uncomplexed lipids, or (iii) some combination of (i) and (ii). 5,12

Our own efforts in this area have focused sharply on the use of the nearest-neighbor recognition (NNR) method for characterizing sterol-phospholipid interactions. ${ }^{13-15}$ In essence, the NNR technique affords molecular-level snapshots of bilayer organization by detecting and quantifying the thermodynamic tendency of exchangeable lipid monomers to become nearest neighbors. ${ }^{15}$ In brief, two lipids of interest, $\mathbf{A}$ and $\mathbf{B}$, are converted into exchangeable dimers (AA, AB and BB), which are then allowed to undergo protomer exchange via thiolate-disulfide interchange. These equilibria are defined by a constant, $K$, which governs the monomer interchange among $\mathbf{A A}, \mathbf{B B}$ and $\mathbf{A B}$ :

$$
\begin{aligned}
& \mathbf{A A}+\mathbf{B B} \stackrel{K}{\rightleftharpoons} 2 \mathbf{A B} \\
& K=[\mathbf{A B}]^{2} /([\mathbf{A A}][\mathbf{B B}])
\end{aligned}
$$


When monomers $\mathbf{A}$ and $\mathbf{B}$ mix ideally, the equilibrium constant takes the value of 4.0. If homoassociations are favored, the equilibrium constant is less than 4.0, whereas favored heteroassociations are characterized by a $\mathrm{K}$ greater than 4.0.

In previous studies, we have shown that, in fluid bilayers made from a "short" exchangeable phospholipid (i.e., 1) and a "longer" analog (i.e., 2), cholesterol induces an apparent preference for homodimer formation (Chart 1). ${ }^{13}$ We have also shown that, in analogous membranes, an exchangeable form of cholesterol strongly favors $\mathbf{2}$ over $\mathbf{1}$ as a nearest-neighbor while it has no effect whatsoever on the ideal mixing of $\mathbf{1}$ and $2 .{ }^{14}$ These findings, together with a growing body of evidence suggesting the formation of cholesterol-phospholipid complexes in fluid monolayers and bilayers, has led us to propose that the cholesterol-induced apparent homodimer preference results from the selective complexation of $\mathbf{2}$ with cholesterol with the result that the complexed $\mathbf{2}$ is withdrawn from participation in the protomer exchange equilibrium. As shown herein, this mechanistic model is testable by a quantitative analysis of the dependency of the equilibrium distribution of exchangeable dimers on the ratio of $\mathbf{1 / 2}$, using a constant cholesterol concentration.

\section{Experimental Section}

\section{Exchangeable Lipids}

Procedures that were used to synthesize the homodimers of $\mathbf{1}$ and $\mathbf{2}$, and the corresponding heterodimer (i.e., $\{\mathbf{1}, \mathbf{1}\},\{\mathbf{2}, \mathbf{2}\}$ and $\{\mathbf{1}, \mathbf{2}\}$, respectively) were similar to those previously described. ${ }^{16}$

\section{Nearest-Neighbor Recognition Measurements}

In a typical liposome preparation, a test tube was charged with a chloroform solution that contained $0.24 \mu \mathrm{mol}$ of $\{\mathbf{1 , 1}\}, 0.36 \mu \mathrm{mol}$ of $\{\mathbf{2 , 2}\}$, and $0.49 \mu \mathrm{mol}$ of cholesterol [or $0.48 \mu \mathrm{mol}$ of $\{\mathbf{1}, \mathbf{2}\}, 0.12 \mu \mathrm{mol}$ of $\{\mathbf{2}, \mathbf{2}\}$ and $0.49 \mu \mathrm{mol}$ of cholesterol]. The chloroform was then evaporated by passing a stream of argon over the solution. The lipid mixture was then dissolved in 100 $\mu \mathrm{L}$ of chloroform and diluted with $270 \mu \mathrm{L}$ of diisopropyl ether. Subsequent addition of $33 \mu \mathrm{L}$ of $3.3 \mathrm{mM}$ tris- $\mathrm{HCl}$ buffer ( $3.3 \mathrm{mM}$ tris- $\mathrm{HCl}, 50 \mathrm{mM} \mathrm{NaCl}, 0.67 \mathrm{mM} \mathrm{NaN}_{3}$ and $0.33 \mathrm{mM}$ EDTA, pH 7.4) produced an emulsion. After the emulsion was sonicated for 3 min, using a mild (bath-type) sonicator, the organic phase was removed by gentle evaporation at $60{ }^{\circ} \mathrm{C}$, resulting in a white gel at the bottom of the test tube. The gel was then collapsed by vigorous vortex mixing for $20-30 \mathrm{~min}$, and $2.0 \mathrm{~mL}$ of additional $10 \mathrm{mM}$ tris- $\mathrm{HCl}$ buffer $(10 \mathrm{mM}$ tris$\mathrm{HCl}, 150 \mathrm{mM} \mathrm{NaCl}, 2 \mathrm{mM} \mathrm{NaN}_{3}$ and $1 \mathrm{mM}$ EDTA, $\mathrm{pH}$ 7.4) was added dropwise with vortex mixing. The dispersion was then degassed with an aspirator for $5 \mathrm{~min}$, and the residual traces of organic solvent removed by dialysis (Spectra/Por Membrane, MWCO 6000-8000) under an argon atmosphere, using three $200 \mathrm{~mL}$ portions of degassed $10 \mathrm{mM}$ tris- $\mathrm{HCl}$ buffer, $\mathrm{pH}$ 7.4) over the course of $18 \mathrm{~h}$. Large unilamellar vesicles formed under these conditions were typically $1000 \mathrm{~nm}$ in diameter (dynamic light scattering).

Thiolate-disulfide interchange reactions were initiated, after the dispersions were equilibrated at $60^{\circ} \mathrm{C}$, by injecting $25.5 \mu \mathrm{L}$ of a tris-buffer solution of $37.65 \mathrm{mM}$ threo-dithiotbreitol ( 0.96 $\mu \mathrm{mol}$ ) and $24 \mu \mathrm{L}$ of a tris-buffer solution, was $0.42 \mu \mathrm{M}$ in monensin (10.2 pmol), with brief vortex mixing, and finally increasing the $\mathrm{pH}$ to 8.5 via addition of ca. $10 \mu \mathrm{L}$ of $1.0 \mathrm{M} \mathrm{NaOH}$. All dispersions were maintained under an argon atmosphere throughout the course of the interchange reactions. Aliquots $(0.30 \mathrm{~mL})$ were withdrawn as a function of time and quenched by addition to a $5.0 \mathrm{~mL}$ test tube containing $85 \mu \mathrm{L}$ of $30 \mathrm{mM} \mathrm{HCl}$ (final $\mathrm{pH} 5.0$ ), followed by brief $(10 \mathrm{~s})$ vortex mixing and immediate cooling to $-20^{\circ} \mathrm{C}$. The frozen samples were then lyophilized and the lipid portion dissolved in $2 \mathrm{~mL}$ of chloroform with vortex mixing for $30 \mathrm{~s}$, followed by centrifugation ( $20 \mathrm{~min}$ ) using a clinical centrifuge. The clear chloroform solution 
was poured into another test tube and chloroform was evaporated under reduced pressure [40 min, 0.4 Torr, $23{ }^{\circ} \mathrm{C}$ ]. The resulting clear film was dissolved in a solution made from $10 \mu \mathrm{L}$ of chloroform plus $90 \mu \mathrm{L}$ of the mobile phase that was used for HPLC analysis. This solution was then immediately analyzed by HPLC using a C18 reverse phase column and a mobile phase that was composed of $10 \mathrm{mM}$ tetrabutylammonium acetate in denatured ethanol/water/ hexane $(82 / 11 / 7, \mathrm{v} / \mathrm{v} / \mathrm{v})$ with flow rate of $0.9 \mathrm{~mL} / \mathrm{min}$. The column was maintained at $31^{\circ} \mathrm{C}$ and the components were monitored at $205 \mathrm{~nm}$ by a Waters 996 -photodiode-array UV detector.

\section{Results and Discussion Complexation Model}

In our model, we assume (i) that cholesterol has negligible affinity towards the homodimer of $\mathbf{1},\{\mathbf{1}, \mathbf{1}\}$, and the heterodimer, $\{\mathbf{1}, \mathbf{2}\}$, (ii) that the homodimer of $\mathbf{2},\{\mathbf{2}, \mathbf{2}\}$ ), is withdrawn from participating in the exchange reaction upon complexation with cholesterol, and (iii) that even in the presence of cholesterol, the equilibrium involving uncomplexed $\{\mathbf{2 , 2}\}$ reacting with $\{\mathbf{1}, \mathbf{1}\}$ to produce two molecules of $\{\mathbf{1 , 2}\}$ is characterized by $K=4.0$. The last assumption may also be expressed as eq. 1 , where $L_{f}$ represents the mol\% of the "longer" homodimer in the uncomplexed state, and $S_{e}$ and $M_{e}$ are the equilibrium mol\% of the "shorter" homodimer and the heterodimer, respectively.

$$
\frac{M_{e}^{2}}{L_{f} \cdot S_{e}}=4
$$

Complex formation then involves the association of cholesterol with $n$ molecules of $L_{f}$ according to eq. 2 . Here, the symbol $C_{f}$ represents the mol\% of uncomplexed cholesterol, $Y$ is the mol\% of the complex that is formed, and $K_{2}$ is a dissociation constant that governs this equilibrium according to eq 3 .

$$
\begin{gathered}
n \cdot L_{f}+C_{j} \underset{K_{2}}{\rightleftarrows} Y \\
K_{2}=\frac{L_{f}^{n} \cdot C_{f}}{Y}
\end{gathered}
$$

Based on experimentally measured values of $M_{e}$ and $S_{e}$, the mol\% of $L_{f}$ can be calculated using eq. 4 , which is a rearranged form of eq. 1 .

$$
L_{f}=\frac{M_{e}^{2}}{4 \cdot S_{e}}
$$

Using the following three stoichiometric relationships, where $L_{e}$ as the total equilibrium mol $\%$ of $\{\mathbf{2 , 2}\}$, and $C_{o}$ as the analytical mol\% of cholesterol that is present in the bilayer, along with eq. 3 , one

$$
\begin{aligned}
& L_{e}=L_{f}+n Y \\
& C_{o}=C_{f}+Y
\end{aligned}
$$

can obtain the following relationship:

$$
K_{2} C_{o}-K_{2} C_{f}=L_{f}^{n} C_{f}
$$

Solving for $C_{f}$ (eq. 5), and then substituting for it yields the relationship that is shown in eq. 6 . 


$$
\begin{gathered}
C_{f}=\frac{C_{o}}{1+\frac{L_{f}^{n}}{K_{2}}} \\
Y=C_{o}-\frac{C_{o}}{1+\frac{L_{f}^{n}}{K_{2}}}
\end{gathered}
$$

One may then express $L_{e}$ as:

$$
L_{e}=L_{f}+n \cdot C_{o}-\frac{n \cdot C_{o}}{1+\frac{L_{f}^{n}}{K_{2}}}
$$

or, alternatively:

$$
L_{e}=L_{f}+\frac{n \cdot C_{o} \cdot L_{f}^{n}}{K_{2}+L_{f}^{n}}
$$

\section{Nearest-Neighbor Recognition Measurements}

Using standard protocols, we measured the dependency of the dimer distribution on the ratio of $\mathbf{1} / \mathbf{2}$ in unilamellar vesicles at $60^{\circ} \mathrm{C}$ in the presence of a fixed concentration of cholesterol; that is, where $C_{o}$ was $44.96 \mathrm{~mol} \%$ in all experiments. ${ }^{17}$ In this work, all mol\%'s are reported in which each lipid dimer is considered as one molecule. Thus, a cholesterol concentration of $44.96 \mathrm{~mol} \%$ corresponds to the nominal concentration of $29 \mathrm{~mol} \%$ if each lipid protomer were regarded as one molecule. ${ }^{17}$ Table 1 lists our results, together with those taken from an earlier study. ${ }^{17}$ In this table, the subscript " $o$ " refers to the composition of the starting reaction mixture and the subscript " $e$ " refers to experimental concentrations that were measured after the exchange reaction has reached equilibrium. As is readily apparent, the complexed form of $\{\mathbf{2 , 2}\}$, namely $\left(L_{e}-L_{f}\right)$, increases more rapidly than $L_{f}$, which indicates that $\mathrm{n}>1$.

A plot of $L_{e}$ versus $L_{f}$ is shown in Figure 1. A nonlinear least squares analysis of these data, based on eq 8 , yielded the best-fit values, $K_{2}=2021 \pm 222 \mathrm{~mol}^{2}$ and $n=2.04 \pm 0.03$. Despite the increase in scatter at low $S_{o}$ values, which is inherent in the experimental method, the data are robust. Thus, if one omits the outlying point at $L_{o}=36.82 \mathrm{~mole} \%$, the analysis yielded the best-fit values $K_{2}=1680 \pm 71 \mathrm{~mol} \%^{2}$ and $n=2.00 \pm 0.03$ (see Supporting Information). Figure 1 also includes the theoretical curve that has been calculated with the best-fit parameters. The excellent agreement between the experimental points and the theoretical curve shows that the results are fully consistent with the proposed mechanism of cholesterol/phospholipid complexation. Of particular relevance is the fact that the value of $n$ is exactly two. This indicates the formation of a true complex of well-defined stoichiometry instead of a mere increase in the number of preferred nearest neighbors.

\section{Discussion}

The phase diagram that has been constructed for mixtures of 1,2-distearoyl-sn-glycero-3phosphocholine (DSPC) and cholesterol indicate that these membranes are fully in the liquidordered phase $\left(l_{o}\right)$ at $29 \mathrm{~mol} \%$ cholesterol and $60^{\circ} \mathrm{C}$; that is, under the conditions used in the present study. ${ }^{18}$ Similary, the phase diagram for mixtures of 1,2-dimyristoyl-sn-glycero-3phosphocholine (DMPC) and cholesterol indicate that such membranes are substantially in the liquid-ordered phase. ${ }^{19}$ If one then considers ternary mixtures of DMPC/DSPC/cholesterol, containing this same cholesterol concentration and variable ratios of DMPC/DSPC, one would expect that these membranes would be dominated by the $l_{o}$ phase at $60^{\circ} \mathrm{C}$. As discussed previously, exchangeable phospholipids such as $\mathbf{1}$ and $\mathbf{2}$ have proven to be excellent mimics of phosphocholines, based on their monolayer properties, melting behavior and their nearest- 
neighbor recognition features in membranes containing variable quantities of cholesterol. ${ }^{20}$ Thus, the experimental system chosen for the present study is a good model for the liquidordered phase of phospholipid bilayers. Given the excellent agreement between our experimental results and our model for complexation, the picture that emerges for this lipid system is as follows: 1) The liquid-ordered phase is homogeneous with all lipids and lipid complexes mixing ideally. 2) A specific complex of cholesterol with four long-chain phospholipids is in rapid equilibrium with free cholesterol and free phospholipid. 3) The complex is fully soluble in the liquid-ordered phase. 4) Cholesterol has a high selectivity for the long-chain phospholipid. 5) Supernumerary cholesterol is freely soluble in this phase up to a high limit. 6) The formation of the liquid-ordered phase creates conditions that are favorable for complex formation, not vice versa.

\section{Conclusions}

This paper shows how nearest-neighbor recognition (NNR) measurements, carried out on cholesterol-rich bilayers containing varying ratios of two exchangeable phospholipids (i.e., 1/ 2), provide strong evidence for the existence of cholesterol/long-chain-phospholipid complexes in the physiologically-relevant fluid phase. These findings strengthen the case for the biological relevance of cholesterol-phospholipid complexes in mammalian membranes rich in cholesterol and high-melting sphingolipids.

\section{Supplementary Material}

Refer to Web version on PubMed Central for supplementary material.

\section{Acknowledgements}

We are grateful to the National Institutes of Health (PHS GM56149) for support of this research.

\section{References}

1. Edidin M. Annu Rev Biomol Struct 2003;32:257-283.

2. Simons K, Vaz WLC. Annu Rev Biomol Struct 2004;33:269-295.

3. Munro S. Cell 2003;115:377-388. [PubMed: 14622593]

4. Veatch SL, Keller SL. Biochim Biophys Acta 2005;1746:172-185. [PubMed: 16043244]

5. McConnell HM, Radhakrishnan A. Biochim Biophys Acta 2003;1610:159-173. [PubMed: 12648771]

6. Feingold, L. Cholesterol in Membrane Models. CRC Press; Ann Arbor: 1993.

7. Vist MR, Davis JH. Biochemistry 1989;29:451. [PubMed: 2302384]

8. Veatch SL, Keller SL. Phys Rev Letters 2002;89:268101.

9. Cao H, Tokutake N, Regen SL. J Am Chem Soc 2003;125:16182-16183. [PubMed: 14692747]

10. Anderson TG, McConnell HM. Biophys J 2001;81:2774-2785. [PubMed: 11606290]

11. Radhakrishnan A, McConnell H. Proc Natl Acad Sci 2005;102:12662-12666. [PubMed: 16120676]

12. (a) Ipsen JH, Karlstrom G, Mouritsen OG, Wennerstrom H, Zuckermann MJ. Biochim Biophys Acta 1987;905:162-172. [PubMed: 3676307] (b) Ipsen JH, Mouritsen OG, Zuckermann MJ. Biophys J 1989;56:661-667. [PubMed: 2819232]

13. Krisovitch S, Liu Y, Regen SL. J Am Chem Soc 1993;115:10104-10110.

14. Sugahara M, Uragami M, Regen SL. J Am Chem Soc 2002;124:4253-4256. [PubMed: 11960454]

15. For a review of the NNR method, see: (a) Davidson SKM, Regen SL. Chem Rev 1997;97:1269-1280. [PubMed: 11851451] (b) Cao H, Tokutake N, Regen SL. J Am Chem Soc 2003;125:16182-16183. [PubMed: 14692747] (c) Sugahara M, Uragami M, Regen SL. J Am Chem Soc 2002;124:4253-4256. [PubMed: 11960454]

16. Cao H, Zhang J, Jing B, Regen SL. J Am Chem Soc 2005;727:8813-8816. [PubMed: 15954788]

17. Dewa T, Miyake Y, Kézdy FJ, Regen SL. Langmuir 2000;16:3735-3739. 
18. Almeida PFF, Vaz WLC, Thompson TE. Biophys J 1993;64:399-412. [PubMed: 8457666]

19. Almeida PFF, Vaz WLC, Thompson TE. Biochemistry 1992;31:6739-6747. [PubMed: 1637810]

20. Zhang J, Cao H, Jing B, Regen SL. J Am Chem Soc 2006;128:265-269. [PubMed: 16390156] 


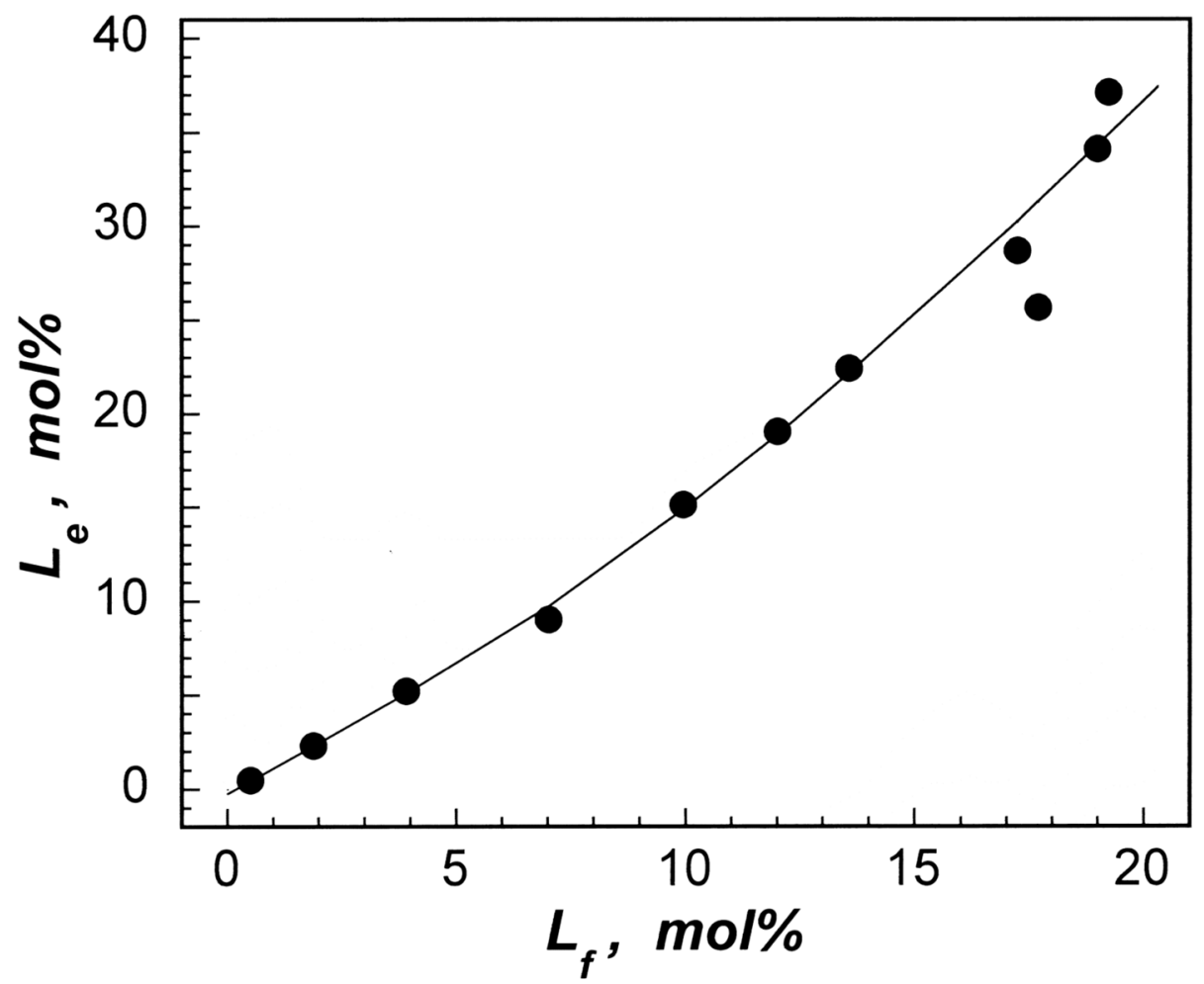

Figure 1.

A plot of $L_{e}$ versus $L_{f}$, data taken from Table 1 . The solid line was calculated using eq 8 with the parameters $K_{2}=2021 \mathrm{~mol}^{2}$ and $n=2.04$. 


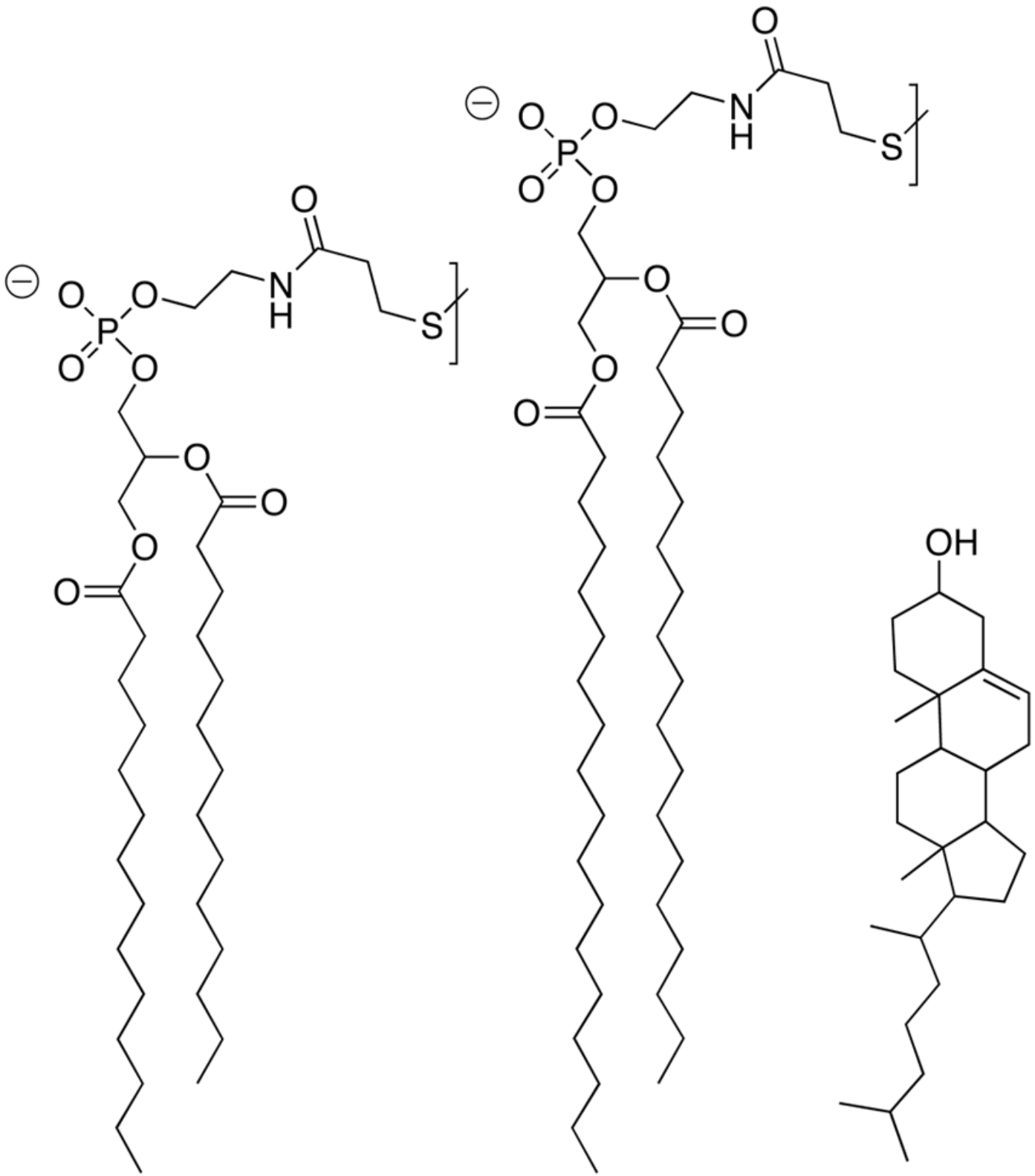

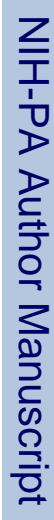

1

2

Cholesterol

Chart 1. 


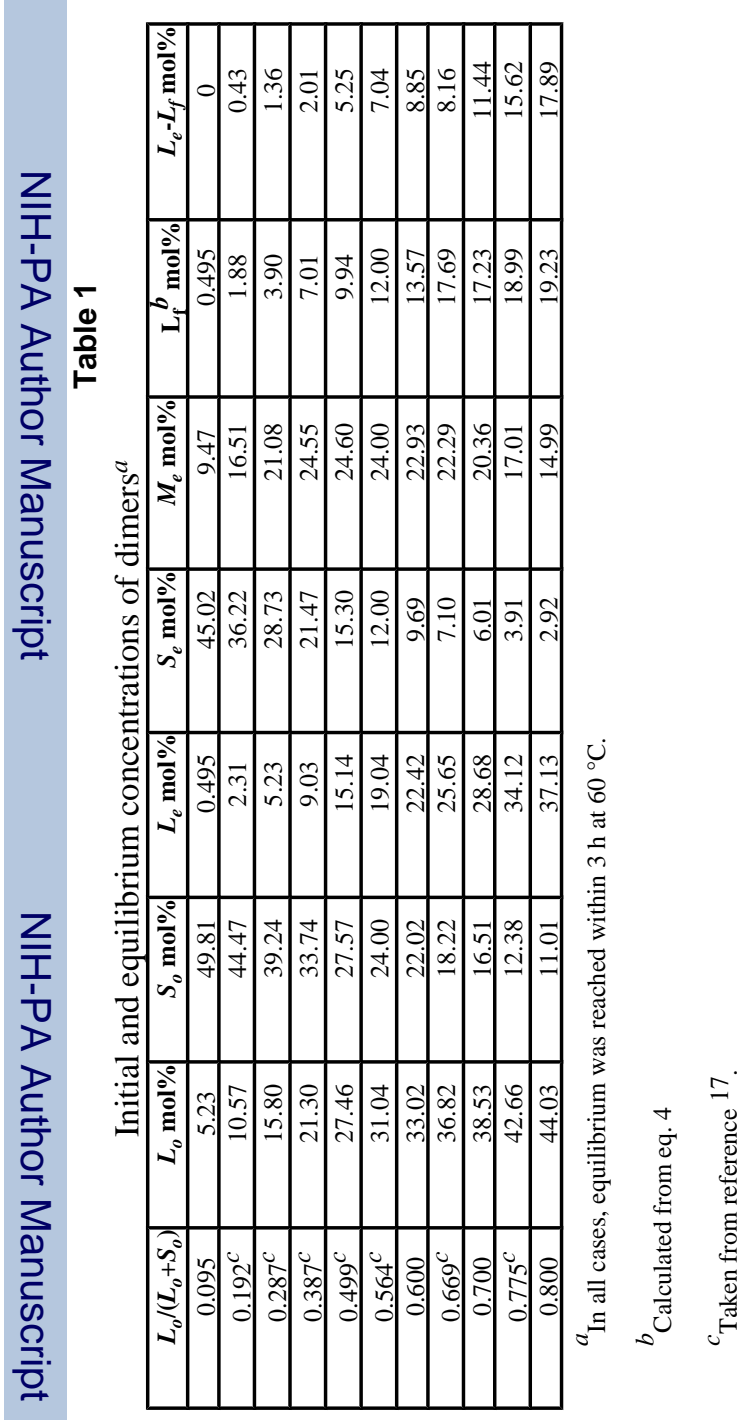

